\title{
Breast implant-associated anaplastic large cell lymphoma: the first South Korean case
}

\author{
Jongwon Lee ${ }^{1}$, Hyungwoo Cho ${ }^{2}$, Dok Hyun Yoon ${ }^{2}$, Eun Key Kim ${ }^{3}$, Gyungyub Gong ${ }^{1}$, Cheolwon Suh ${ }^{2}$, Joo-ryung Huh ${ }^{1}$ \\ Departments of ${ }^{1}$ Pathology, ${ }^{2}$ Oncology, and ${ }^{3}$ Plastic Surgery, Asan Medical Center, University of Ulsan College of Medicine, Seoul, Korea
}

To this date, breast implant-associated anaplastic large cell lymphoma (BIA-ALCL) has seldom been reported in the Asian population, despite the not insignificant number of breast implant surgeries performed locally. Here, we report the first case of BIA-ALCL in South Korea.

\section{CASE REPORT}

A 47-year-old woman presented to a local clinic who had previously undergone bilateral breast augmentation via transaxillary subpectoral augmentation (TSA) in 2013 for cosmetic reasons. The implanted device was a Natrelle silicone-filled textured breast implant (style 115, BIOCELL Textured Round Midrange Projection Gel-filled Breast Implant, $253 \mathrm{~mL}$, Allergan, Dublin, Ireland). Six years postoperatively, in 2019, she noticed swelling of her right breast. An ultrasonographic examination revealed fluid collection and capsule discontinuity, suggesting a ruptured implant (Fig. 1A). On magnetic resonance imaging, however, implants were found intact, though fluid collection was prominent in the inner posterior side of the right breast (Fig. 1B).

Under the impression of implant rupture with seroma, aspiration was performed revealing large, atypical lymphocytes with irregular kidney-shaped nuclei, with occasional binucleation (Fig. 2A). These cells were confirmed as atypical T-cells positive for $\mathrm{CD} 30$ and $\mathrm{CD} 3$ and negative for anaplastic lymphoma kinase 1 per immunostaining, a typical phenotype of anaplastic lymphoma kinase (ALK)-negative BIA-ALCL (Fig. 2B, C) [1].

Whole-body positron-emission tomography (PET) showed

Received: April 28, 2020 Revised: June 30, 2020

Accepted: July 1, 2020

Corresponding Author: Joo-ryung Huh, MD

Department of Pathology, Asan Medical Center, University of Ulsan College of

Medicine, 88 Olympic-ro 43-gil, Songpa-gu, Seoul 05505, Korea

Tel: +82-2-3010-4553, Fax: +82-2-472-7898, E-mail: jrhuh@amc.seoul.kr hypermetabolic capsule and fluid collection confined to the right breast. Blood test results were normal and bone marrow biopsy findings were negative for lymphoma involvement. With curative intent, the bilateral implants were removed with capsulectomy, which revealed irregular thickening of the capsule taken from the right breast with multiple focal areas of fibrinous deposits in the lining without distinct tumor masses (Fig. 3A). The implant did not show grossly identifiable foci of rupture. The capsule and implant from the left breast were unremarkable.

According to histologic sectioning, large atypical lymphocytes positive for $\mathrm{CD} 30$ but negative for ALK immunostaining that had infiltrated the luminal surface of the capsule from the right breast (Fig. 3B, C) were consistent with pathologic stage T2. Focal foreign body-type macrophages were identified throughout the specimen, denoting microscopic leakage (Fig. 3D). T-cell clonality was detected on gene rearrangement study for beta T-cell and gamma T-cell receptor genes. The contralateral breast was unremarkable, with a thin capsule and the absence of atypical lymphocytes.

\section{DISCUSSION}

BIA-ALCL is a rare type of T-cell lymphoma, occurring in recipients of either saline or silicone-filled textured type breast implants. BIA-ALCL is usually localized and the prognosis is excellent. Complete surgical removal of the capsule and implant with negative resection margins is curative in almost all patients. However, advanced-stage disease with metastasis has also been reported [2], which emphasizes the importance of the awareness of the entity and the making of an early diagnosis.

BIA-ALCL most commonly presents as effusion around the implant simulating rupture of the implant, while tumor mass formation is less frequent. The present case to our knowledge is

\section{}



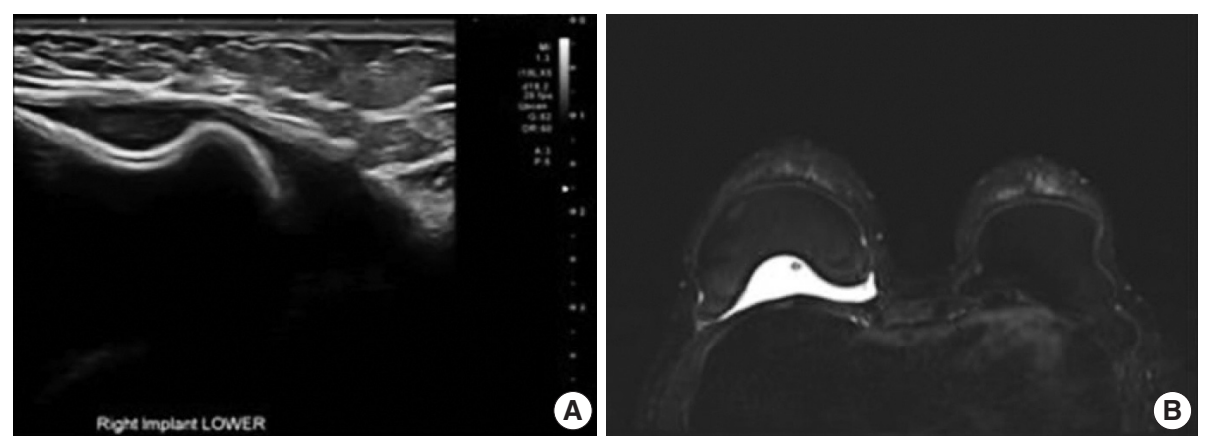

Fig. 1. Imaging studies. (A) Ultrasonography of the right breast shows suspected inner capsule discontinuity due to fluid collection, suspicious for rupture. (B) Magnetic resonance imaging of the breasts. Fat-suppressed T2-weighted axial image and dynamic contrast-enhanced T1-weighted axial image were processed. Fluid collection was identified at the inner side of the right breast capsule.
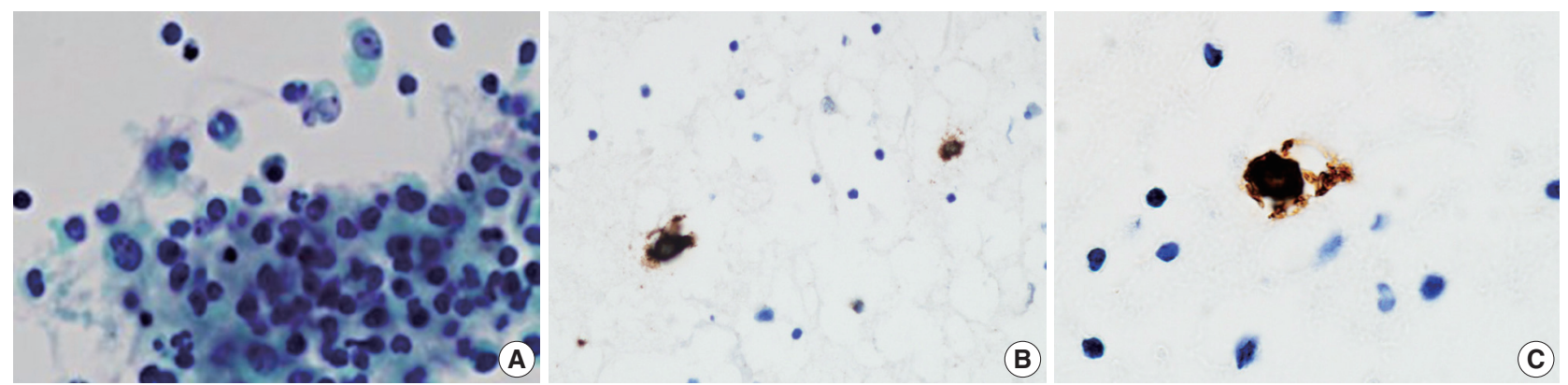

Fig. 2. (A) Large lymphocytes with binucleation and horseshoe-like nuclear indentation are visible. Tumor cells are admixed with mature, small lymphocytes for size comparison (SurePath Papanicolaou stain, Becton, Dickinson and Company, Franklin Lakes, NJ, USA). (B) Large atypical cells positive for CD30 are admixed with mature lymphocytes. (C) Large neoplastic cells with membranous, Golgi staining pattern of CD30 immunolabeling.
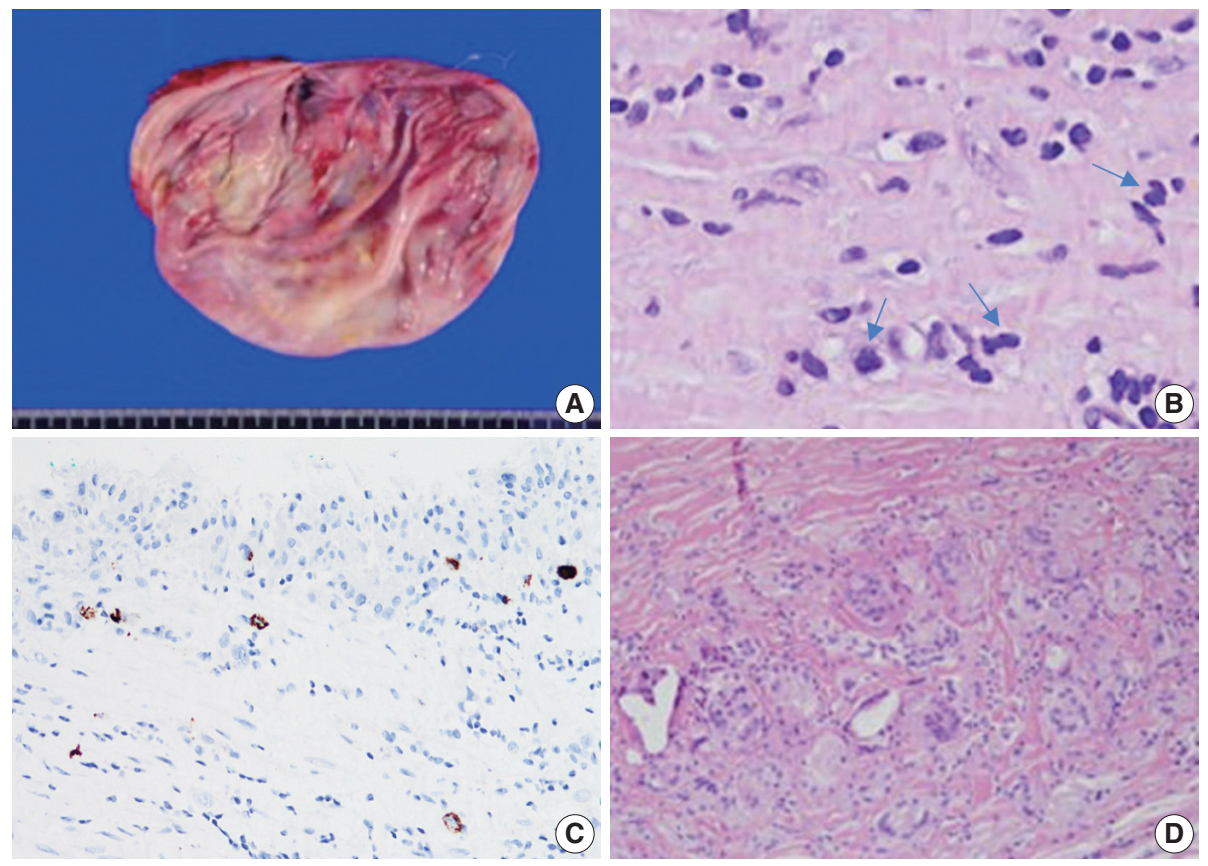

Fig. 3. The capsule removed from the right breast. (A) The capsule appeared irregularly thickened without definite "tumor" masses though with irregular, fibrin-like deposits in multiple foci. (B) Microscopically, atypical large lymphocytes with indented, kidney-shaped nuclei (arrows) had infiltrated the fibrinous material on the luminal side of the capsule. (C) The same cells show diffuse CD30 immunopositivity. (D) Foreign body-type giant cells were gathered around the deep surface of the capsule. 
the first report of BIA-ALCL in a South Korean patient, with the typical presentation being similar to that in Western countries both clinically and pathologically. With 27,393 annual Allergan breast prostheses implanted in South Korea alone, one would expect a higher number of cases, so the apparent rarity of BIA-ALCL may be due to a trend of underdiagnosis. Due to the low index of suspicion, aspiration cytology is rarely performed in Korea for implant-associated effusions (personal observation, E.K.K.). The present case should raise a clinical awareness that BIA-ALCL does arise in Korean women and investigation to rule out BIA-ALCL should be performed in appropriate cases.

\section{Ethics Statement}

This case was deemed exempt by the Asan Medical Center Institutional Review Board (IRB \#2020-0433). Informed consent was obtained from individual participant included in this study.

\section{ORCID}

Jongwon Lee https://orcid.org/0000-0003-3057-7874

Hyungwoo Cho https://orcid.org/0000-0003-4307-2795

Dok Hyun Yoon https://orcid.org/0000-0002-8289-3548

Eun Key Kim
Gyungyub Gong https://orcid.org/0000-0001-5743-0712

Cheolwon Suh https://orcid.org/0000-0002-9178-4431

Joo-ryung Huh https://orcid.org/0000-0002-6991-6397

\section{Author Contributions}

Conceptualization: JRH, JL, CS. Data curation: EKK, HC, DHY, JRH, JL. Methodology: JRH, JL, GG. Writing—original draft: JRH, JL. Writing—review \& editing: JRH, JL. Approval of final manuscript: all authors.

\section{Conflicts of Interest}

G.G., a contributing editor of the Journal of Pathology and Translational Medicine, was not involved in the editorial evaluation or decision to publish this article. All remaining authors have declared no conflicts of interest.

\section{Funding Statement}

No funding to declare.

\section{References}

1. Di Napoli A, Pepe G, Giarnieri E, et al. Cytological diagnostic features of late breast implant seromas: from reactive to anaplastic large cell lymphoma. PLoS One 2017; 12: e0181097.

2. Quesada AE, Medeiros LJ, Clemens MW, Ferrufino-Schmidt MC, Pina-Oviedo S, Miranda RN. Breast implant-associated anaplastic large cell lymphoma: a review. Mod Pathol 2019; 32: 166-88. 